\title{
DETERMINING RELATIVE ADEQUACY IN BIBLICAL INTERPRETATION
}

\author{
Ernst M Conradie \\ Department of Religion and Theology \\ University of the Western Cape, \\ Louis C Jonker \\ Department of Old and New Testament \\ Stellenbosch University
}

\section{Introduction}

Plurality is an inevitable and necessary result of Biblical interpretation. There can be no single fully adequate interpretation of any Biblical text. This is partly due to the polisemy inherent in the text itself, but also to the different historical, geographical, and ecclesial contexts within which the meaning of a text is continuously being appropriated. Interpretation is necessarily an ongoing task if this implies the need to embody the significance of the text in and for ever changing circumstances.

This plurality is amply illustrated in the recent history of Biblical hermeneutics, with its emphasis on the conflicting diversity of witnesses within the Biblical canon, with its uncovering of numerous factors influencing Biblical interpretation and in the continued emergence of new approaches to Biblical interpretation itself.

Any post-modern appreciation for this plurality has to acknowledge that not all interpretations could be regarded as adequate. Some interpretations clearly have to be resisted fiercely and fearlessly. To recognise corrupt interpretations is perhaps far easier than to provide a full account of adequate interpretation. Yet the search for relatively adequate interpretation cannot be avoided. This requires some criteria to distinguish adequate from less adequate interpretations. But where can such criteria be found? How can appropriate criteria be defined if any such criteria are also open to an interpretative plurality? Who will judge any particular interpretation on the basis of such criteria? With what authority and legislative power? With which interests? And for whom? The rhetoric of these questions is clear. However, the need for the discernment, judgement and, if necessary, resistance of inadequate interpretations cannot be avoided.

In this contribution a strategy is proposed to test and develop the relative adequacy of specific Biblical interpretations. However, the following should be admitted right from the start about the criteria suggested here: These criteria or questions do not have a constitutive function, but rather a critical function. This implies that the application of these criteria will not necessarily constitute adequate interpretation. They do not necessarily lead to "better" interpretation. The criteria may rather serve as a "checklist-in-retrospect", as tools that may be used in the quest for adequacy in Biblical interpretation.

1. Cf. also Conradie et al $(1995,267)$ : "The hermenutic detour has at least made us aware of the numerous factors that can play a role in the interpretation of the Bible. It provided no method that can be rigorously implemented to ensure adequate interpretation, rather it cultivated a sensitivity to the variables that may play a role. At the same time it made us aware of the dangers that plague the interpretation of the Bible. This at least heightens the possibility of reaching more adequate interpretations. The difference between a relatively more 
This remark also implies that these criteria are not presented to Bible study groups or individual readers of the Bible with the suggestion that they will help these readers to interpret the Bible "better" ${ }^{2}$. The criteria are rather presented to Biblical and hermeneutical scholars with the hope that they will help these scholars to analyse the process of interpretation "better".

\section{The context: a process of critical testing}

The context within which the search for relative adequacy in Biblical interpretation is situated, requires further comment. All too often debates on relative adequacy still focus on the "point of departure" of a particular interpretation. In the rhetoric and rivalry amongst scholars one can hear pleas to start with the regula fidei, the magisterium, the creeds and confessions, a surface reading of the Greek and Hebrew texts, the narrative structure, genre, style and rhetoric of the text, the literary history of the text itself, the socio-historic forces behind the text, a pastoral response to the questions people ask about their faith, the spirituality of the faith community embodying the text, the needs of the poor, oppressed and marginalised, the ideologies of sexism, racism, and classism infiltrating the reception of the Biblical texts, etc.

This list illustrates that such a point of departure is highly contested, that several positions are feasible and that the choice of a point of departure cannot guarantee and cannot be used to determine relative adequacy. Relative adequacy is thus not a function of the point of departure.

The argument of this contribution is that relative adequacy is a function of the degree of critical testing that a particular interpretation can endure (Popper). The notion of critical testing is derived from a model for interpretation that was proposed in the publications Fishing for Jonah ${ }^{3}$ and Angling for interpretation. ${ }^{4}$ This model suggests that readers can only start from where they are at a given moment. The following three phases may thus be distinguished in the process of interpretation, coinciding with the well-known spiral of interpretation:

Phase 1: An attempt to articulate one's pre-understanding (or immediate understanding) of a text, the way in which one is currently interpreting a text;

Phase 2: A process of continuous critical testing;

Phase 3: The renewal of this existing (tradition of) interpretation.

In any Bible study group all three these phases may be identified during the course of a Bible study event. The various group members articulate their views on the text and allow the text and the other members in the group to inform, challenge and enrich their views. This leads to a constant renewal of existing interpretations of the particular text as the Bible study event proceeds.

or relatively less adequate interpretation is extremely hard to articulate - perhaps because interpretation requires artistry rather than a method. The difference can be discussed only with the aid of metphors."

2. Hermeneutical reflection by means of these criteria may even lead to negative side effects. The following side effects were discussed in Conradie et al (1995, 262ff.): (a) A further fragmentation of interpretation rather than a wealth of meaning; (b) Critical distance replaces active engagement or vice versa; (c) Sterility replaces theological complacency; and (d) Exclusivity and "privatization" replace critical dialogue.

3. Conradie et al (1995, especially section A5).

4. Conradie \& Jonker (2001, especially chapter 5). 
In a Bible study group the process of critical testing does not take place in a particularly disciplined or systematic way. By contrast, the discipline of hermeneutics allows for a more detailed and systematic process of critical testing in which various aspects of a particular interpretation may be related to a wide variety of other insights bearing on the interpretation of the particular text. In scholarly discourse a rich array of insights from a wide range of scholarly disciplines may be drawn upon for this purpose. The argument of this contribution is that the descriptive tools that were developed in the research project on empirical Biblical hermeneutics may be used to structure this process of critical testing and to identify criteria that may be used in this regard.

It should be stressed, though, that such critical testing cannot guarantee adequacy in interpretation. Indeed, critical testing has a critical and not a constitutive function. This also applies to the way in which scholars engage in the critical testing of the interpretations of the Bible in Bible study groups. Such critical testing may not lead to a renewal of existing interpretations in the Bible study groups since this would depend on the interaction between a particular Bible study group and the scholarly discourse. The process of critical testing may at least help to identify crude inadequacies in a given interpretation. However, the process of critical testing itself is open to ideological abuse. The process of critical testing can therefore, at best, take place in the form of an ongoing conversation in which the criteria for evaluating a particular interpretation are themselves open to debate.

\section{Criteria for determining relative adequacy in Biblical interpretation}

If critical testing is regarded as a dialogue with other perspectives, who should the conversation partners be? What should the agenda for the conversation be? What critical questions should be asked in order to determine the relative adequacy of a particular interpretation? These questions call for criteria that can assist us to determine relative adequacy in Biblical interpretation.

The openness of the ongoing hermeneutical conversation about interpretations does not prevent the identification of a particular agenda for the conversation - as long as the agenda itself remains open for negotiation. The identification of the various criteria discussed below may at least provide an agenda to guide the ongoing conversation about relative adequacy, but should not be regarded as prescriptive or restrictive.

\section{a) Biblical interpretation as communication}

In Jonker's contribution in this volume on the different "maps" that have been proposed to distinguish between all the factors that influence Biblical interpretation, it has already emerged that many of these maps take some sort of a communication model as presupposition. With regards to Biblical interpretation the most basic representation of the communication process may be illustrated as follows:

5. See the "Preface on empirical Biblical hermeneutics in this volume for a more detailed discussion of this problem. 


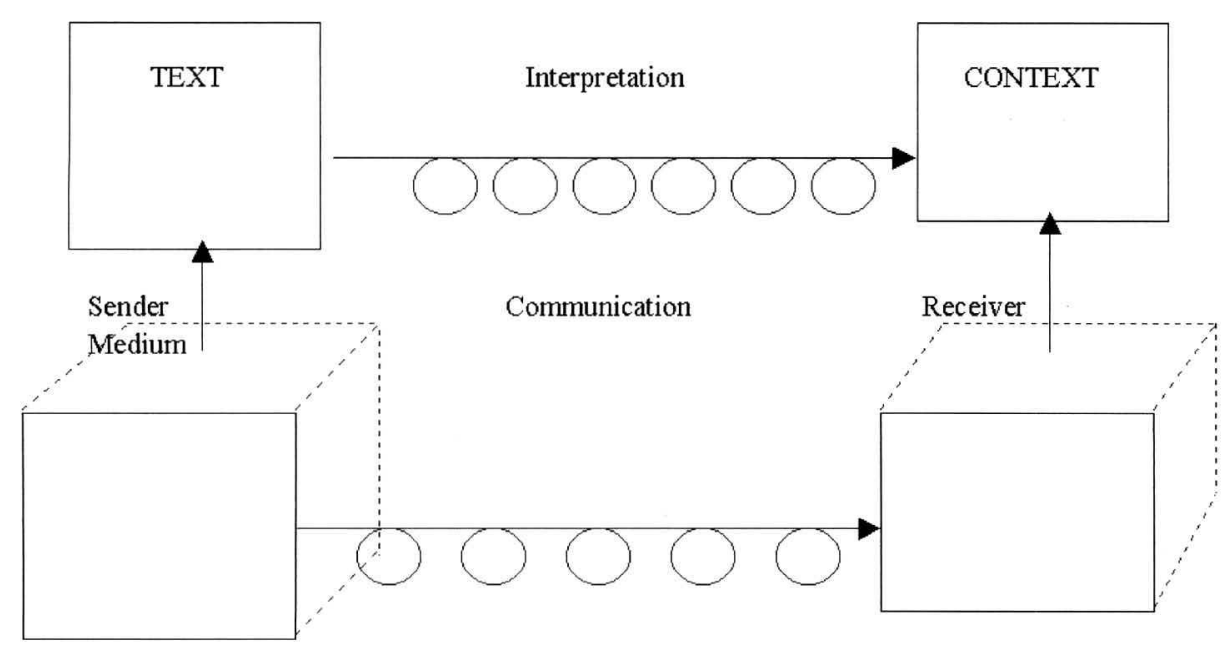

\section{Diagramme 1}

The basis of this representation is a distinction between Text and Context, which corresponds with a distinction between Sender/Medium ${ }^{6}$ and Receiver. Communication implies that the Sender/Medium should be related to the Receiver, or, that the Text has to be related to the Context. The gap between Text and Context therefore has to be bridged, or (to use Gadamer's language) a fusion of horizons has to take place.

To determine the adequacy of interpretation according to this representation, it would be necessary to assess to what extent the fusion of horizons has actually taken place. This presents us with at least three criteria for determining relative adequacy:

- Does the interpretation fully involve the horizon of the text?

- Does the interpretation fully involve the horizon of the context?

- What strategies are used to relate these horizons with one another?

Daniel Patte ${ }^{7}$ has proposed the distinction between legitimacy and validity in determining the success of the communication between text and context. According to him a legitimate interpretation is an interpretation that is based on actual dimensions of the text, while the validity of an interpretation is determined by the specific cultural, religious and social circumstances in which the interpretation takes place. This implies that there may be more than one legitimate interpretation (depending on the dimensions of the text being incorporated in the interpretation) of a given text. Likewise, there may different

6. The combination of Sender and Medium in the Text, is the result of the Biblical texts being ancient texts. The sender (oral tradents, authors, compositors and redactors) can only be known / investigated through and by means of the medium, the text.

7. In an unpublished paper delivered at the SBL International Meeting in Vienna in 1990. A more elaborated description of Patte's contribution is provided in Conradie et al $(1995,38)$. 
interpretations that are valid in different contexts. An interpretation that is valid in a given context may be invalid in another context. One should even reckon with the possibility that an illegitimate interpretation (that is, an interpretation that is not based on dimensions in the text) may be valid in a specific context of interpretation.

Four positions can therefore be distinguished:

- An interpretation may be legitimate, but not valid;

- An interpretation may be legitimate and valid;

- An interpretation may be illegitimate, and also not valid;

- An interpretation may be illegitimate, but nevertheless valid.

According to the criteria proposed by Patte, an adequate interpretation would then be an interpretation that is both legitimate and valid.

\section{b) Further refinement: Seven sets of criteria}

In terms of some of the other "maps discussed in Jonker's contribution in this volume, the criteria of legitimacy and validity are not the only criteria that may be identified for adequate Biblical interpretation. The basic distinction that has been discussed in the previous section, namely the distinction between text and context, can be complemented with some other aspects as shown in Diagramme $2{ }^{8}$ This allows for a further refinement of various criteria for relative adequacy

c) Tradition of interpretation

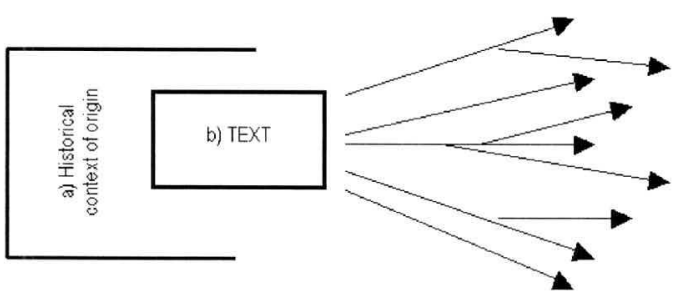

d) Spiral of ongoing interpretation

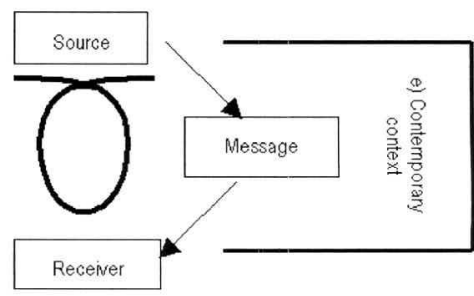

g) In its rhetorical context

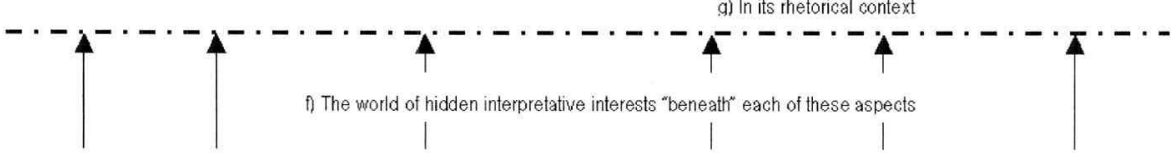

\section{Diagramme 2}

On this basis, at least the following seven criteria (with associated questions) may be identified for relatively adequate Biblical interpretation (The numbering of the paragraphs below corresponds to the letters in the diagram):

a) Historical consciousness:

To what extent is an interpretation sensitive to the historical distance between the world in which the text originates and the contemporary context? Does an interpretation take

8. Cf. also the more elaborated description in Conradie \& Jonker (2001, chapter 4). 
account of the way in which various "hands" cooperated in the production of a particular text? Does an interpretation take account of the particular historical, sociocultural, political, economic and religious circumstances that played a role in the process of textual production?

b) Legitimacy:

To what extent does an interpretation take the textual structures (be they grammatical, rhetorical or narratological) into account? Does an interpretation take account of the genre of the text? Does it respond appropriately to this genre? Does an interpretation take account of the immediate literary context of the text under discussion? Does an interpretation take account of the broader (canonical) context of a particular text?

c) Fruitfulness:

To what extent does an interpretation enable a particular tradition of interpretation "to continue" "? How does an interpretation relate to and respond to the beliefs, praxis, customs, ethos, values, projects, institutions of a particular tradition, e.g. an ecclesial tradition, a faith community, or a scholarly guild? Does it repeat, reinforce, or challenge these aspects? Is the interpretation innovative in the sense that it will yield fruitful new possibilities for the self-understanding of the community's identity? What is the transformative potential of such innovations? Will the interpretation really lead to new forms of praxis? Is an interpretation perhaps too innovative in the sense that it can no longer be recognised by that tradition / community as authentic?

d) Sophistication:

What doctrinal keys and interpretative strategies ${ }^{10}$ are used in the particular interpretation? To what extent does these interpretative strategies and doctrinal keys take account of more than one similarity between text and context and also of the differences between text and context? How do the doctrinal keys relate to the systems of symbols in the tradition of interpretation? Do the dominant doctrinal keys manage to do justice to the complexities of both the text and the relevant context? Or do they distort both text and context in an attempt to harmonise the differences?

e) Relevance / Validity:

To what extent does an interpretation respond to a thorough analysis of the social, economic and political dimensions of a particular contemporary context? Does an interpretation reflect anything about the historical, socio-cultural, political, economic and religious circumstances in which it is situated? Does it take the problems, needs and questions that arise from these circumstances into account? Does the interpretation tend to reduce the contemporary context to only one trait? Does it take the complexities of a social context into account?

9. This requires both a degree of continuity and of discontinuity with respect to the tradition of interpretation (ecclesial, confessional, theological, hermeneutical) within which an interpreter is situated. The adherents to this tradition should be able to recognise their heritage in the interpretation and to identify the interpretation as an appropriate innovation of this tradition.

10. For a discussion of these terms, see the contribution of Conradie elsewhere in this volume. 
f) Openness and inclusiveness:

To what extent is an interpretation free from ideological distortion? ${ }^{11}$ Can any bias (e.g. male or patriarchal interests) be detected in the rhetoric of the text itself? Does an interpretation deliberately select or suppress any factor that may play a significant role in the interpretation of a particular text? Can any trace of the influence of specific rules of conduct, expectations, influential leaders, role models, social codes, conventions and power interests be detected in a particular interpretation? Does psychological dispositions, economic interests, or any particular ideology (e.g. racism, classism, sexism, elitism, colonialism) perhaps distort the interpretation in any way?

\section{g) Rhetorical efficacy:}

What effect does the interpretation aim at in the particular context in which the text is interpreted? Who is saying what to whom with this interpretation? To whom is the interpretation communicated explicitly and / or implicitly? How was it communicated? To what extent did the medium of communication influence the message? How did the group dynamics influence the rhetorical aim?

It would be possible to refine these criteria even further. ${ }^{12}$ However, an overelaborate refinement of criteria will not necessarily enhance the thoroughness of the ongoing conversation on relative adequacy.

\section{c) Dialogue with interpretations}

These criteria can, of course, not be used in any rigid or instrumental way to evaluate or even "measure" the relative adequacy of a given interpretation. How do they then contribute to the process of determining relative adequacy in interpretation?

It has already been mentioned above that the process of critical testing presupposes dialogue. The criteria formulate an agenda for this dialogue as part of the scholarly endeavour to determine relative adequacy. The criteria remind scholars of a variety of perspectives that may, at times, influence the process of interpretation. They help us to ask relevant questions in the search for relative adequacy.

If the proposed criteria may function as an agenda for the ongoing conversation on relative adequacy, it should be added that this agenda does not prescribe a specific order of

11. This has to be tested in conversation with ideology-critical analyses, being sensitive to the possibility of pervasive distortions through the ideologies of inter alia classism, colonialism, sexism, elitism and anthropocentrism.

12. For example, in the project on "Intercultural Bible reading" mentioned above, a provisional set of 13 criteria was defined as guidelines for the description of interpretations. Apart from the seven criteria mentioned in this article, the following are also included in the project guidelines: (i) Strategies of imagination (How are gaps in the text filled in?); (ii) 'Reading with whom' (With which character(s) in the text did the reader associate?); (iii) Appropriation strategies (What model was used for the appropriation of meaning?); (iv) Praxeological effect (Did the group develop new praxes from their understanding of the text?); (v) Reading attitude (What is the attitude driving the reader to interpret the text?). Furthermore, what is described under "Sophistication" in our description above, was split up in the project guidelines into "Interpretational strategies" and "Codes / heuristic keys".

Although this distinction of criteria is also quite helpful, it seems that some of the criteria overlap and do not really deal with separate issues. For example, the "appropriation strategies" distinguished in the guidelines do not really differ from the "interpretational strategies" (discussed under "sophistication" in our description above). 
discussion. Not every conversation on relative adequacy needs to cover the full agenda either. $^{13}$ The configuration of criteria that are present in a particular conversation will depend on many factors, inter alia on the genre of the interpreted text ${ }^{14}$ and on the group dynamics.

\section{Conclusion}

The quest for relatively adequate Biblical interpretation and for criteria to test relative adequacy remains a crucial but precarious task. The criteria identified in this contribution are equally precarious. They suggest a possible agenda for a more structured conversation on relatively adequacy in Biblical interpretation. However, the agenda clearly remains open for further negotiations.

\section{BILBIOGRAPHY}

Conradie, EM et al 1995. Fishing for Jonah. Various approaches to Biblical interpretation. Bellville: UWC Publications.

Conradie, EM \& Jonker, LC 2001. Angling for interpretation. A guide to understand the Bible better. Bellville: UWC Publications.

13. Cf. Jonker in Conradie et al $(1995,274)$ : "I can imagine that some people will hear us saying, "the more conversation partners, the merrier'. This was not quite the object of our exercise in interpretation though. We tried only to indicate possible conversation partners. In the interpretation of a particular text some conversation partners may be silent or even absent."

14. Poetic texts, for example, tend to be to some extent "timeless" and "universal", and do not always require a great deal of investigation into the world of origin of the texts.

15. If an interpretation is the result of a group with a strong leader, it is often difficult to discern whether the interpretation gives evidence of the leader's context, doctrinal keys and interpretative strategies, or rather of the group as a whole. 